\title{
Theta Rhythmic Stimulation of Stratum Lacunosum-Moleculare in Rat Hippocampus Contributes to Associative LTP at a Phase Offset in Stratum Radiatum
}

\author{
Sarah J. Judge and Michael E. Hasselmo \\ Department of Psychology Center for Memory and Brain, Program in Neuroscience and Center for BioDynamics, \\ Boston University, Boston, Massachusetts 02215
}

Submitted 29 August 2003; accepted in final form 2 May 2004

\begin{abstract}
Judge, Sarah J. and Michael E. Hasselmo. Theta rhythmic stimulation of stratum lacunosum-moleculare in rat hippocampus contributes to associative LTP at a phase offset in stratum radiatum. J Neurophysiol 92: 1615-1624, 2004. First published May 5, 2004; 10.1152/jn.00848.2003. Computational modeling demonstrates that encoding and context-dependent retrieval of memories in region CA1 of the hippocampus will be most effective when the phase of strongest entorhinal input (to stratum lacunosum-moleculare) is offset from the phase of maximal induction of long-term potentiation at Schaffer collateral synapses (in s. radiatum). This would allow entorhinal input to play a role in both retrieval and encoding without engaging long-term potentiation (LTP) during retrieval. Experiments in brain slice preparations of the hippocampal formation tested the relationship between rhythmic input to s. lacunosum-moleculare and the time of maximal LTP induction at Schaffer collateral synapses in s. radiatum. Analysis of the data demonstrates a statistically significant difference in the induction of LTP for different time intervals between the end of each four-pulse train in s. lacunosum-moleculare and the single pulse s. radiatum stimulation. The time of maximal LTP induction was found to be $\sim 30 \mathrm{~ms}$ after the end of lacunosum-moleculare stimulation, consistent with the requirements of the model.
\end{abstract}

\section{N T R O D U C T I O N}

The hippocampal theta rhythm is a $3-$ to $10-\mathrm{Hz}$ oscillation that appears in electrencephalographic (EEG) recordings from the hippocampus of rats during active sniffing, exploration, and rapid-eye-movement (REM) sleep (Bland 1986; Buzsaki 2002; Green and Arduini 1954). Theta rhythm appears to be correlated with the effectiveness of memory function in specific tasks (Berry and Thompson 1978; Seager et al. 2002; Winson 1978), and theta rhythm appears to phase lock with specific components of memory tasks (Givens 1996; Macrides et al. 1982). Within each cycle of theta, the synaptic properties of neurons in the hippocampus change, including phasic differences in the induction of long-term potentiation (LTP) at specific synaptic connections. The induction of LTP was shown to depend on the phase of stimulation at the synapses of the perforant pathway terminating in the molecular layer of the dentate gyrus in anesthetized rats initially (Pavlides et al. 1988), and this effect was later demonstrated in awake behaving rats (Orr et al. 2001). Similar phase sensitivity appears at the synapses of the Schaffer collaterals (SC) arising from region CA3 pyramidal neurons and terminating on the dendrites of region CA1 pyramidal cells in stratum radiatum. LTP

Address for reprint requests and other correspondence: M. Hasselmo: Dept. of Psychology, Boston University, 2 Cummington St., Boston, MA 02215 (E-mail: hasselmo@bu.edu). induction at these synapses has been shown to be maximal with stimulation on the positive phase of theta rhythm recorded locally both in vivo (Hölscher et al. 1997; Hyman et al. 2003) and in vitro (Huerta and Lisman 1995). In these studies, stimulation at the trough of local CA1 theta induces LTD or depotentiation (Huerta and Lisman 1995; Hölscher et al. 1997; Hyman et al. 2003). Note that these studies in CA1 all involved recording of theta rhythm locally in stratum radiatum (referred to as CA1 theta) rather than more distally at the hippocampal fissure, which is the standard reference location for most studies of theta rhythm in whole animal preparations (e.g., Fox et al. 1986).

Current-source-density analysis has demonstrated that synaptic currents in different layers of the hippocampus change phasically with theta (Bragin et al. 1995; Brankack et al. 1993; Buzsaki et al. 1986). As noted in the preceding text, most studies use theta rhythm recorded at the hippocampal fissure as the standard reference phase for hippocampal theta. The hippocampal fissure lies between stratum lacunosum-moleculare of region CA1 and the molecular layer of the dentate gyrus. At the trough of the theta rhythm recorded at the hippocampal fissure, current sinks in s. lacunosum-moleculare are strong (Brankack et al. 1993), indicating strong excitatory synaptic currents at the synapses of perforant path fibers which arise from the entorhinal cortex (EC) (Witter et al. 1988). At this same phase, there is a current source in stratum radiatum (Brankack et al. 1993), indicating that there is little synaptic transmission at Schaffer collateral synapses that arise from CA3 (see Amaral and Witter 1989). In contrast, at the opposite phase (the peak) of CA1 fissure theta, synaptic currents in s. lacunosum-moleculare are weak, but s. radiatum currents are strong (Brankack et al. 1993).

A recent hippocampal model (Hasselmo et al. 2002a,b) reveals how the changes in the synaptic properties in the hippocampus during each theta cycle could provide separate functional phases for encoding and retrieval. The features of this model are summarized in Fig. 1. In this model, encoding of new associations takes place during the time period around the trough of CA1 fissure theta. This is close to the peak of theta recorded locally in s. radiatum; this correlates with stronger induction of LTP in s. radiatum (Hölscher et al. 1997; Huerta and Lisman 1995; Hyman et al. 2003). This is also the phase when EC input is strongest, allowing a strong response to

\footnotetext{
The costs of publication of this article were defrayed in part by the payment of page charges. The article must therefore be hereby marked "advertisement" in accordance with 18 U.S.C. Section 1734 solely to indicate this fact.
} 


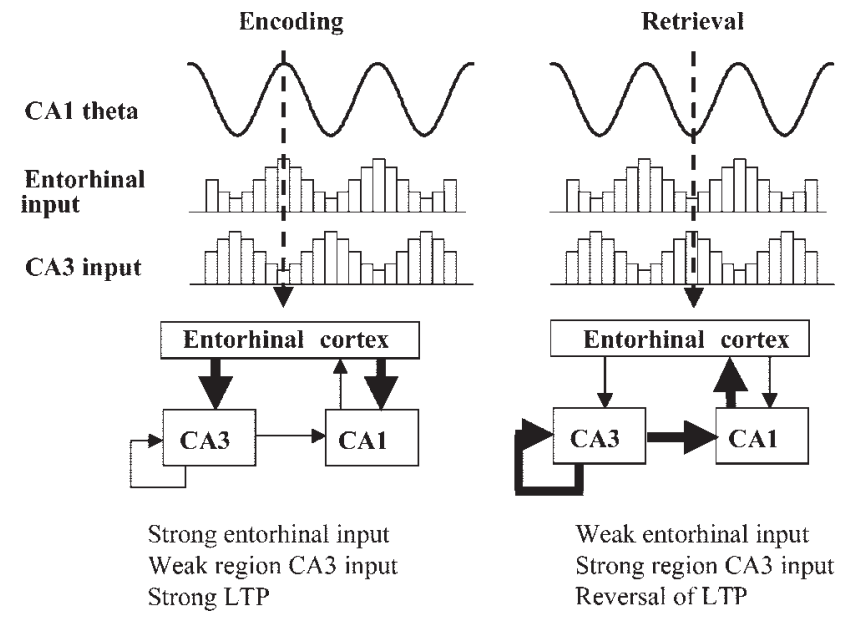

FIG. 1. Schematic representation of the change in dynamics during hippocampal theta. Left: dynamics for encoding. At the peak of local CA1 theta, synaptic currents arising from entorhinal cortex are strong. Synaptic transmission arising from CA3 is weak but the same synapses show a strong capacity for long-term potentiation (LTP), allowing effective encoding of new associations, while preventing interference from prior associations. Right: dynamics for retrieval. At the trough of CA1 theta, synaptic currents arising from entorhinal cortex are relatively weak. Synaptic transmission arising from CA3 is strong but the same synapses undergo depotentiation rather than LTP. This allows effective retrieval of prior associations. Note that CA1 theta is out of phase with fissure theta.

afferent input. At this phase, synaptic inputs arising from CA3 are weakest, preventing interference from prior associations. Together these effects provide effective dynamics for encoding. The model proposes that retrieval of previously stored associations occurs on a separate phase of each theta cycle, during the time period around the peak of fissure theta. During this phase of theta, physiological properties are appropriate for retrieval. EC input in s. lacunosum-moleculare is weak, but synaptic currents in s. radiatum caused by Schaffer collateral input from region CA3 are strong, allowing retrieval of associations stored by previous modification of the Schaffer collaterals. Consistent with this retrieval function, burst stimulation at this phase of theta does not cause LTP in s. radiatum of CA1 (Hölscher et al. 1997; Hyman et al. 2003). This absence of LTP could allow retrieval of old associations without causing interference with new learning.

The previously published models of theta rhythm function (Hasselmo et al. 2002a,b) focused on retrieval mediated by the spread of activity across Schaffer collateral synapses in s. radiatum. However, the entorhinal input may play an important role in regulating context-dependent retrieval from region CA1. The analysis presented here extends the previous model by showing that if the entorhinal cortex plays a role in contextdependent retrieval, then the phase of entorhinal input should be slightly different from the phase of LTP induction.

Most previous studies of LTP induction during theta rhythm (Hölscher et al. 1997; Huerta and Lisman 1995; Hyman et al. 2003) would not demonstrate an offset between phase of LTP induction and phase of entorhinal input because they primarily study LTP induction at two distinct phases of theta rhythm (peak and trough), partly due to difficulties in precisely regulating the time of stimulation presentation relative to an endogenous theta rhythm. Thus those studies do not explore intermediate values that could show stronger or weaker induc- tion or a complex function differing from a sine wave or a square wave.

In contrast, slice studies with dual stimulation location allow detailed analysis of the LTP induced with different delays between the stimulation of entorhinal input fibers in s. lacunosum-moleculare and CA3 input in s. radiatum. Previous studies using this technique have shown that the timing of s. lacunosum-moleculare stimulation (EC input) relative to s. radiatum stimulation (CA3 input) modulates LTP (Levy et al. 1998; Remondes and Schuman 2002). LTP at SC-CA1 pyramidal neuron synapses in $\mathrm{s}$. radiatum is reduced when $\mathrm{s}$. radiatum conditioning trains, which can induce LTP alone, are both preceded and followed by s. lacunosum-moleculare conditioning trains (Levy et al. 1998; Remondes and Schuman 2002). However, when the s. radiatum trains are delayed to coincide with the end of s. lacunosum-moleculare stimulation, LTP is not inhibited (Levy et al. 1998). Note that other earlier studies showed that the relative timing of dual inputs in s. radiatum alone determine the induction of LTP versus long-term depression (LTD) (Stanton 1996; Stanton and Sejnowski 1989).

These previous slice studies show the importance of the relationship between the $\mathrm{EC}$ input and $\mathrm{CA} 3$ input in the induction of LTP but do not study a wide range of delays. To analyze the possible relationship between rhythmic synaptic input and LTP induction, we investigated how synaptic plasticity in s. radiatum is modulated by the timing of s. radiatum stimulation relative to the phase of rhythmic stimulation of $s$. lacunosum-moleculare at theta frequency. The timing of the $s$. radiatum stimulation, in relation to the s. lacunosum-moleculare stimulation, was varied for each experiment in the range of 0-195 ms to analyze the changes in synaptic input strength. In addition, the level of s. radiatum stimulation used in this study was ineffective at inducing LTP alone (single pulses at $5 \mathrm{~Hz}$ ) so the influence of s. lacunosum-moleculare stimulation on LTP induction could be examined, unlike in previous studies (Levy et al. 1998; Remondes and Schuman 2002). A preliminary account of these results has been previously published in abstract form (Judge and Hasselmo 2002).

\section{METHODS}

\section{Slice preparation}

Hippocampal slices were prepared from female Sprague-Dawley rats (2-6 wk old) by standard procedures (Hasselmo and Schnell 1994). After light anesthetization with halothane, the rats were decapitated. The brains were removed quickly and immersed in ice-cold oxygenated cutting solution with the following composition (in $\mathrm{mM}$ ) 248 sucrose, $2.5 \mathrm{KCl}, 1.3 \mathrm{MgSO}_{4}, 10$ dextrose, $26 \mathrm{NaHCO}_{3}, 1.2$ $\mathrm{KH}_{2} \mathrm{PO}_{4}$, and $2.4 \mathrm{CaCl}_{2}$. The dorsal surface of the brain was glued to the chilled stage of a vibratome against an Agar block and covered in ice-cold oxygenated cutting solution. Slices (350 $\mu \mathrm{m}$ thick) were cut in the horizontal plane. Extraneous cortical tissue was gently dissected away to give two hippocampal slices from each horizontal slice. The slices were incubated at room temperature in a submersion-type chamber containing oxygenated artificial cerebrospinal fluid (ASCF) with the following composition (in $\mathrm{mM}$ ) $124 \mathrm{NaCl}, 2.5 \mathrm{KCl}, 1.3$ $\mathrm{MgSO}_{4}, 10$ dextrose, $26 \mathrm{NaHCO}_{3}, 1.2 \mathrm{KH}_{2} \mathrm{PO}_{4}$, and $2.4 \mathrm{CaCl}_{2}$. After $\geq 1 \mathrm{~h}$ of incubation, slices were placed in a submerged recording chamber perfused with oxygenated artificial cerebrospinal fluid (ASCF; $34 \pm 0.5^{\circ} \mathrm{C}$; mean $\pm \mathrm{SE}$ ) at $4 \mathrm{ml} / \mathrm{min}$. Extracellular recordings were made with glass micropipettes of $\sim 1 \mathrm{M} \Omega$ impedance filled with $2 \mathrm{M} \mathrm{NaCl}$. A single-recording electrode was positioned in $\mathrm{s}$. 
radiatum $\sim 100 \mu \mathrm{m}$ away from s. lacunosum-moleculare to record the Schaffer collateral-evoked excitatory postsynaptic potentials (EPSPs) and monitor the potentials evoked by s. lacunosum-moleculare stimulation. Stimulation was delivered through a Neurodata PG4000 digital stimulator with two bipolar tungsten electrodes. One stereotrode was placed in s. radiatum to stimulate the Schaffer collaterals, and the other was placed on the opposite side of the recording electrode in s. lacunosum-moleculare to stimulate the entorhinal cortex fibers. Electrode positions in the hippocampal slice preparation are illustrated in Fig. $2 A$.

\section{Experimental protocol}

Each experiment consisted of a stimulation protocol (Fig. 2B), which was preceded and followed by a test period. During the test periods, single $100-\mu$ s test pulses $(<100 \mu \mathrm{A})$ were delivered to $\mathrm{s}$. radiatum every $30 \mathrm{~s}$. Test pulse EPSPs were required to have an amplitude of $0.6 \mathrm{mV}$ without signs of a population spike. The strength of the test pulse was adjusted to evoke a response amplitude that was $\sim 50 \%$ of the maximum response. After the amplitude of the test pulse EPSPs had stabilized (i.e., did not change for $\geq 15 \mathrm{~min}$ ) and the baseline amplitude was determined, the stimulation protocol was delivered. S. lacunosum-moleculare was stimulated with a strong (200 $\mu \mathrm{A})$ theta-burst pattern $(10$ bursts at $5 \mathrm{~Hz}, 4$ pulses per burst at 100 $\mathrm{Hz}$ ), which closely resembles the firing pattern of layer III entorhinal cortical neurons recorded in vivo (Chrobak and Buzsaki 1998; Chrobak et al. 2000). Note that this stimulation approximates the phasic nature of excitatory and feedforward inhibitory synaptic input to s. lacunosum-moleculare during theta rhythm but does not replicate many other modulatory effects within the hippocampus regulated by other inputs such as the medial septum. S. radiatum was stimulated with single pulses at $5 \mathrm{~Hz}$ set at the same stimulus intensity used
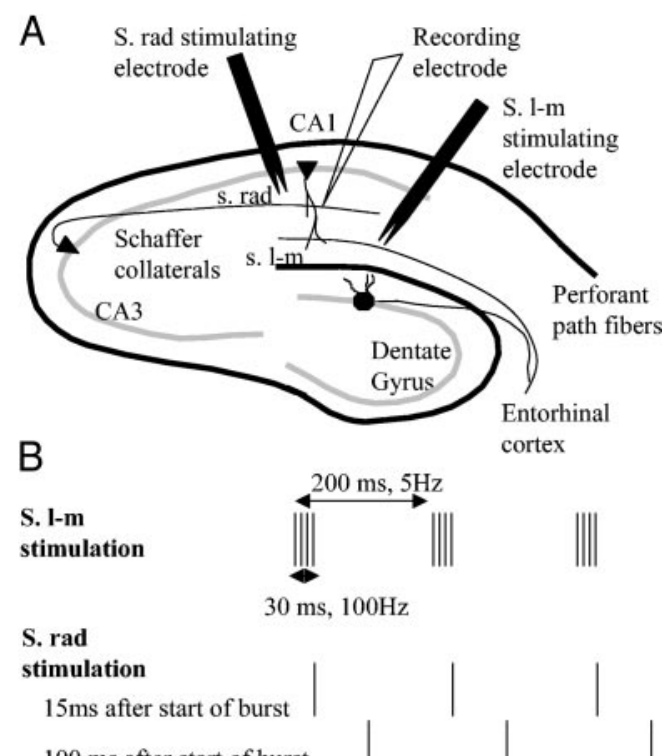

$100 \mathrm{~ms}$ after start of burst

FIG. 2. A: diagram of electrode positions in CA1 region of hippocampal slice preparation. One stimulating stereotrode was positioned in stratum radiatum (s. rad) among Schaffer collateral fibers arising from CA3 and another was positioned in s. lacunosum-moleculare (s. 1-m) among perforant path fibers arising from the entorhinal cortex. An extracellular recording electrode was placed in s. radiatum. $B$ : Schematic diagram of stimulation protocol delivered to the slice. 10 bursts of 4 pulses $(100 \mathrm{~Hz}, 200 \mu \mathrm{A})$ at $5 \mathrm{~Hz}$ were delivered to s. lacunosum-moleculare. Single pulses at $5 \mathrm{~Hz}$ were delivered to $\mathrm{s}$. radiatum. The timing of the s. radiatum pulses in relation to the s. lacunosum-moleculare bursts was varied for each experiment. The relationship between the s. radiatum pulse and the s. lacunosum-moleculare burst is schematically shown for 15 and $100 \mathrm{~ms}$ after the start of the s. lacunosummoleculare burst. during the test period. The timing of the s. radiatum pulses in relation to the s. lacunosum-moleculare bursts during the stimulation protocol was varied for each experiment $(5,15,25,40,50,60,70,80,100,110$, $120,130,140,150,160,180,190$, or $195 \mathrm{~ms}$ after the start of the s. lacunosum-moleculare burst). Note that these individual delays can also be considered as number of degrees within the full cycle of 200 $\mathrm{ms}$. Therefore in this framework, $0 \mathrm{~ms}$ would be $0^{\circ}$. However, note that the actual peak of dendritic depolarization caused by the stimulation in s. lacunosum-moleculare would fall at or just after the last of the four stimulus pulses. This is a reasonable assumption for the peak of the oscillation of entorhinal input. Therefore we will usually consider $0^{\circ}=30 \mathrm{~ms}$. The reference oscillation shown in the figures has $0^{\circ}$ at $30 \mathrm{~ms}$. In this case, $80 \mathrm{~ms}=90^{\circ}$ and $130 \mathrm{~ms}=180^{\circ}$.

\section{Analysis of experimental data}

Amplified responses were collected and stored by a computer using Clampex software (Axon Instruments). The peak negative-deflection of the EPSPs was measured from the individual recordings taken at 30 -s intervals during the test periods. The responses to test pulses after the stimulation protocol were expressed as a percentage of the prestimulation protocol baseline response. Results are listed as means \pm SE taken $30 \mathrm{~min}$ after the stimulation protocol unless otherwise stated. Traces are single sweeps.

\section{Mathematical analysis of theta phase relationships}

The mathematical analysis used here builds on previously published models (Hasselmo et al. 2002a,b), which model the encoding and retrieval of associations between two spatial locations or between one spatial location and reward. The storage of associations in these models depends on Hebbian synaptic modification of the synapses of Schaffer collateral fibers arising in region CA3 and terminating in s. radiatum of region CA1. The LTP tested in these experiments provides a means of testing the magnitude of synaptic modification at these synapses.

The performance of these models is analyzed using a mathematical performance measure (Hasselmo et al. 2002a,b), which tests how much a retrieved memory pattern resembles the desired pattern that was stored minus its resemblance to other patterns that could interfere with the desired pattern. This performance measure allows evaluation of the accuracy of retrieval based on the spread of activity during encoding and retrieval and the modification of Schaffer collateral synapses. The full details of this derivation are provided in a previous paper (Hasselmo et al. 2002b) available at http://people.bu.edu/hasselmo/HasselmoHayIlynGorch.pdf. Here we will start with the results of the analysis from that previous paper, specifically with the performance measure presented in Eq. 2.18 of that paper as follows

$$
\begin{aligned}
M=\int_{0}^{m 2 \pi} \theta_{\text {somaCAl }}(t) & \theta_{\mathrm{ECIII}}(t) \mathrm{d} t+\int_{0}^{m 2 \pi} \theta_{\mathrm{somaCAl}}(t) \theta_{\mathrm{CA} 3}(t) \mathrm{d} t \\
& \times\left\{\int_{0}^{n 2 \pi} \theta_{\mathrm{LTP}}(t)\left[\theta_{\mathrm{ECIII}}(t)\right] \mathrm{d} t-\int_{0}^{n 2 \pi} \theta_{\mathrm{LTP}}(t)\left[\theta_{\mathrm{CA} 3}(t)\right] \mathrm{d} t\right\}
\end{aligned}
$$

In this equation, $M$ is the performance measure, and the individual functions $\theta$ refer to functions representing sinusoid oscillations in specific physiological variables that have been shown to oscillate during theta rhythm. Specifically, the function $\theta_{\text {LTP }}$ refers to the oscillation in the ease of induction of LTP in s. radiatum (Hölscher et al. 1997; Hyman et al. 2003). The function $\theta_{\text {ECIII }}$ refers to the oscillation in magnitude of entorhinal input to s. lacunosum-moleculare as demonstrated using current-source-density analysis (Brankack et al. 1993). The function $\theta_{\mathrm{CA} 3}$ refers to the oscillation in magnitude of CA3 input to s. radiatum also shown by current-source-density data (Brankack et al. 1993). The function $\theta_{\text {somaCA1 }}$ refers to oscillations in 
the depolarization of pyramidal cells in region CA1 as shown with intracellular recording (Fox 1989; Kamondi et al. 1998). In this paper, we are focusing on the difference in phase between the rhythmic entorhinal input to s. lacunosum-moleculare, represented by $\theta_{\mathrm{ECIII}}$, and the induction of LTP in s. radiatum., represented by $\theta_{\mathrm{LTP}}$. The performance measure depends on the multiplicative interaction of these functions computed over an integer number of oscillations (as represented by the integrals with $2 \pi$ multiplied by the integer $n$ for number of cycles during initial behavioral interaction with the stimulus and $m$ for the number of cycles during which behavioral retrieval is being tested).

The individual oscillatory functions are simple sine waves that have been shifted algebraically to stay positive. Each sine wave function $\theta$ is associated with a specific phase parameter $\phi$ that determines the phase difference in the peak of each function from a reference point (this phase difference can be described as the number of degrees between the peaks). Thus the function $\theta_{\mathrm{ECIII}}$ is associated with a phase constant $\phi_{\text {ECIII }}$, which determines where its peak will occur relative to other oscillating functions. For example, if $\phi_{\mathrm{ECIII}}$ is 90 and $\phi_{\mathrm{LTP}}$ is 120 , then the phase of maximal LTP induction is delayed relative to the entorhinal input by a phase difference of $30^{\circ}$. The sine wave functions all have the same equation differing only by these phase parameters. Thus for example, the function $\theta_{\mathrm{ECIII}}$ takes the form

$$
\theta_{\mathrm{ECIII}}(t)=1 / 2 * \sin \left(t+\phi_{\mathrm{ECIII}}\right)+1 / 2
$$

The solution obtained by integration of $E q .1$ will be described in RESULTS. The integral solution to $E q .1$ was implemented in MATLAB 6.5 , allowing computation of the performance measure with a range of different values of the phase parameters $\phi_{\mathrm{ECIII}}, \phi_{\mathrm{CA} 3}$, and $\phi_{\mathrm{somaCA} 1}$. This was done while holding the phase constant at zero $\left(\phi_{\mathrm{LTP}}=0\right)$ for the LTP phase parameter. The other phase parameters were altered by $10^{\circ}$ steps, allowing determination of the phase parameters for maximal performance.

\section{RES U L T S}

\section{Mathematical analysis}

The mathematical analysis demonstrates that best performance of the model occurs when the peak of the induction of LTP $\theta_{\text {LTP }}$ is delayed by $\sim 30^{\circ}$ relative to the peak of entorhinal input $\theta_{\mathrm{ECIII}}$. This differs from the result described in previous papers (Hasselmo et al. 2002a,b), where the best performance is obtained with zero degree phase difference between $\theta_{\mathrm{EC}}$ and $\theta_{\text {LTP. }}$ This difference occurs because previously published papers on this model assumed that the oscillation in induction of long-term potentiation could go positive and negative, as a standard sine wave $(E q .3)$, whereas the result presented here constraints LTP to remain positive (as shown in Eq. 4)

$$
\begin{gathered}
\text { Previous papers: } \theta_{\mathrm{LTP}}(t)=\sin \left(t+\phi_{\mathrm{LTP}}\right) \\
\text { Current paper: } \theta_{\mathrm{LTP}}(t)=1 / 2 * \sin \left(t+\phi_{\mathrm{LTP}}\right)+1 / 2
\end{gathered}
$$

In the previous papers, this resulted in the maximum value of the performance measure being obtained when the phase of entorhinal input and LTP had zero phase difference $\left(\theta_{\mathrm{EC}}=\right.$ $\theta_{\mathrm{LTP}}=0^{\circ}$ ) and when CA3 input and postsynaptic depolarization were exactly out of phase with the other functions (that is, $\theta_{\mathrm{CA} 3}$ and $\left.\theta_{\text {somaCA1 }}=180^{\circ}\right)$. However, the assumption in previous papers that LTP would go positive and negative resulted in the best function being obtained when interfering memories were completely forgotten. This is not consistent with data on actual memory function where an interfering memory can be retrieved in a different context. Here we allow memories to persist in the model despite interference by assuming that LTP stays positive, as in Eq. 4. If we make this assumption, the integral solution to Eq. 1 takes the form

$$
\begin{aligned}
M=(1 / 4) \pi\left\{\left[\cos \left(\phi_{\mathrm{somaCA}}-\phi_{\mathrm{ECIII}}\right)+2\right]+(1 / 4) \pi\left[\cos \left(\phi_{\mathrm{somaCA} 1}-\phi_{\mathrm{CA} 3}\right)+2\right]\right. \\
\left.\times\left[\cos \left(\phi_{\mathrm{LTP}}-\phi_{\mathrm{EC}}\right)-\cos \left(\phi_{\mathrm{LTP}}-\phi_{\mathrm{CA} 3}\right)\right]\right\}
\end{aligned}
$$

Computing this performance measure with a reference point of $\phi_{\mathrm{LTP}}=0^{\circ}$ gives a notion of how performance varies based on the relative values of LTP and EC. Finding the maximum of the performance measure at a resolution $10^{\circ}$ demonstrates that the best function is found at $\phi_{\mathrm{EC}}=30^{\circ}$ and $\phi_{\mathrm{somaCA} 1}=100^{\circ}$ (with the additive form of the phase parameter $\phi_{\mathrm{EC}}$ used in $E q$. 2 , a positive value means the phase $\phi_{\mathrm{EC}}=30^{\circ}$ comes earlier than the LTP oscillation with $\phi_{\text {LTP }}=0$ ). Note that this estimate is based on a sine wave modulation, which means that there will be a significant range of phases during which induction of LTP will be strong. Thus induction of LTP will be strong over a broad range of values $\sim 30^{\circ}$ after the EC peak, taking values $>50 \%$ of the peak at delays between -60 and $120^{\circ}$ after the EC peak. Thus the distribution of phase values is relatively broad in the model, but the location of this distribution can be described by referring to the center of the distribution at the peak of the sine wave $\left(30^{\circ}\right)$. Note that similarly, if the difference between EC and LTP differs from $30^{\circ}$, the performance of the network degrades slowly for values close to $30^{\circ}$. The function of performance for different phase differences has a sine wave shape (shifted relative to 0 ). If performance at a $30^{\circ}$ phase difference is $100 \%$, then the performance is at $90 \%$ of this value for phase relationships between 357 and $63^{\circ}$. This distribution will be compared with the induction of LTP at a range of phase differences between EC and CA3 stimulation in the physiological data presented next. Curve fitting with the data allows determination of the approximate center of the distribution of LTP induction in the data; this allows an overall comparison of the distribution in the model and the data.

\section{Experimental data}

In these experiments, robust LTP was consistently observed in slices dependent on the relative timing of stimulation. The induction of LTP, as measured by the change in the amplitude of test pulse EPSPs before and after the stimulation protocol, was dependent on the timing of the s. radiatum pulse in relation to the s. lacunosum-moleculare burst during the stimulation protocol. The test pulse EPSP was potentiated when s. radiatum was stimulated at phases following closely after s. lacunosum-moleculare stimulation (Fig. 3A) and depressed when s. radiatum was out of phase with the s. lacunosum-moleculare (Fig. 3B).

Stimulation of s. radiatum within the $30-\mathrm{ms}$ s. lacunosummoleculare bursts during the stimulation protocol potentiated the test pulse EPSP amplitude by $24 \pm 8.4 \%$ (15 ms after burst start; $n=5$ ). Note that because it is exactly halfway through the s. lacunosum-moleculare burst, this could be considered to be before the peak of EC input $(-15 \mathrm{~ms}$ before burst end or $-27^{\circ}$ before 0 phase). The test pulse EPSP was also potentiated when the s. radiatum pulses were delivered within $100 \mathrm{~ms}$ of the start of the s. lacunosum-moleculare bursts (that is, within $70 \mathrm{~ms}$ or $126^{\circ}$ after the end of the s. lacunosum-moleculare 


\section{A Control 2 minutes $\quad 10$ minutes 30 minutes}

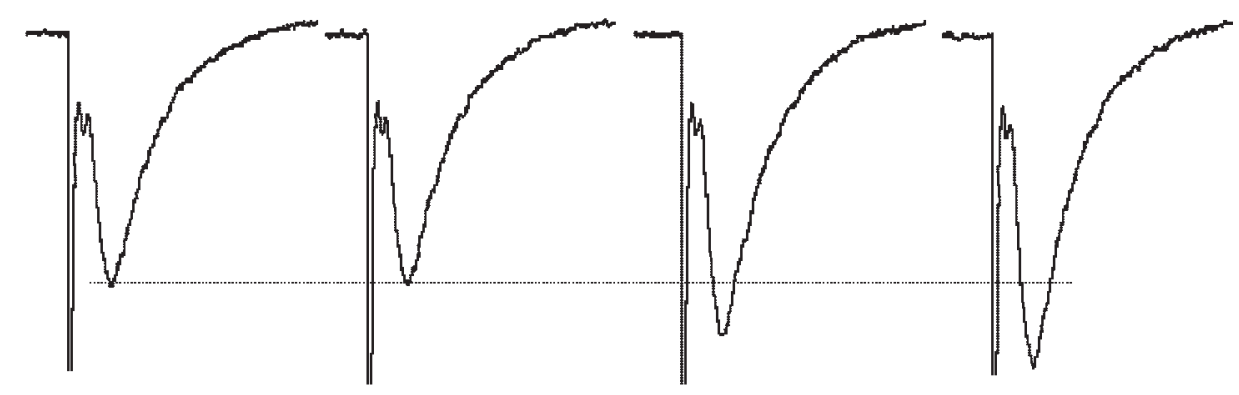

B

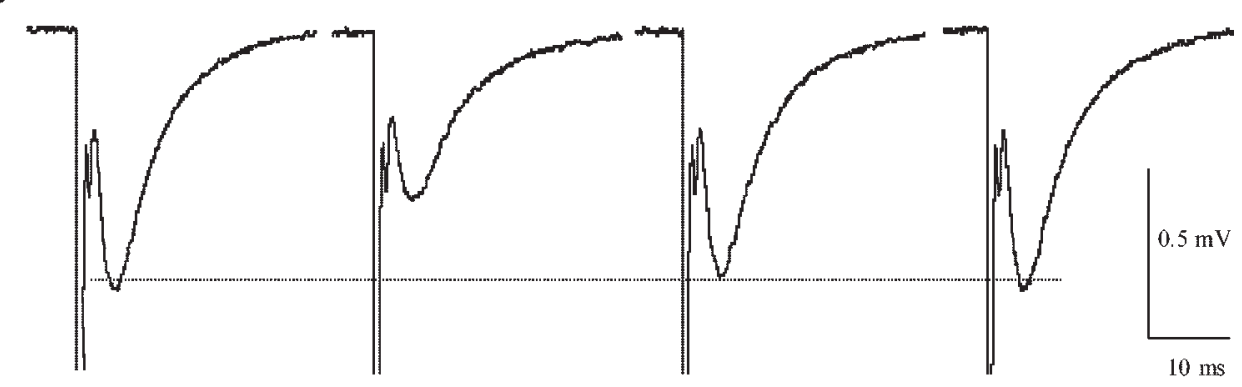

FIG. 3. Examples of individual excitatory postsynaptic potentials (EPSPs) recorded in s. radiatum in response to Schaffer collateral stimulation before and at different time points after the stimulation protocol. $A$ : test pulse EPSP traces from an experiment in which s. radiatum was stimulated $60 \mathrm{~ms}$ after the start of the s. lacunosum-moleculare burst $\left(30 \mathrm{~ms}\right.$ or $54^{\circ}$ after the end of the s. lacunosum-moleculare burst) during the stimulation protocol. The test pulse amplitude is unchanged 2 min after the stimulation protocol but is potentiated after $10 \mathrm{~min}$ and remains potentiated after $30 \mathrm{~min}$. B: test pulse EPSP traces from an experiment in which s. radiatum was stimulated $160 \mathrm{~ms}$ after the start of the s. lacunosum-moleculare burst $\left(130 \mathrm{~ms}\right.$ or $234^{\circ}$ after the end of the $\mathrm{s}$. lacunosum-moleculare burst) during the stimulation protocol. The test pulse amplitude is depressed 2 min after the stimulation protocol, but this is short term as the EPSP returns to control amplitude within10 min of the stimulation protocol. burst) during the stimulation protocol. S. radiatum pulses delivered 40, 60, and $100 \mathrm{~ms}$ after the start of the s. lacunosummoleculare bursts, potentiated the test pulse EPSP by $+25 \pm$ $6.7 \%(n=6),+27 \pm 6 \%(n=2)$, and $+27.7 \pm 3.7 \%(n=2)$, respectively (Fig. $3 A$ ). Considered in relation to the end of the burst, these fall $10 \mathrm{~ms}\left(18^{\circ}\right), 30 \mathrm{~ms}\left(54^{\circ}\right)$, or $70 \mathrm{~ms}\left(126^{\circ}\right)$ after the end of the burst. Figure $4 A$ summarizes the mean results for all experiments based on recordings obtained $30 \mathrm{~min}$ after the stimulation protocol. The time course of synaptic modification in this figure is fitted to a sine wave plotted in Fig. $4 A$. In addition to this sine wave fitted to the LTP induction data, the figure also shows the timing of s. lacunosum-moleculare stimulation pulses at the bottom.

In contrast to the induction of LTP with small phase delays, longer phase delays did not allow induction of LTP as shown in Fig. 4A. Stimulation of s. radiatum 110-195 ms after the start of the s. lacunosum-moleculare bursts $(80-165 \mathrm{~ms}$ or 144 to $297^{\circ}$ after the end) during the stimulation protocol produced no significant change in the test pulse EPSP amplitude obtained at $30 \mathrm{~min}$ (mean percentage EPSP change $=-0.5 \pm 0.5150$ ms after burst start, $n=2 ;+7 \pm 4160 \mathrm{~ms}$ after burst start, $n=$ $2 ;+3.5 \pm 0.5180 \mathrm{~ms}$ after burst start, $n=2)$. Therefore LTP was only induced when s. radiatum was stimulated within 100 ms of the start of the s. lacunosum-moleculare bursts during the stimulation protocol (that is, $70 \mathrm{~ms}$ or $126^{\circ}$ from the end of the burst)

As shown in Fig. $4 B$, examination of the test pulse EPSPs within a few minutes of the stimulation protocol revealed a short-term depression that was induced when s. radiatum pulses were delivered 110-195 ms after the start of the s. lacunosum-moleculare bursts during the stimulation protocol. For example, s. radiatum pulses delivered 120, 160, and 180 ms after the start of the s. lacunosum-moleculare bursts, depressed the test pulse EPSP by $-23 \pm 6.5 \%(n=2),-24.5 \pm$ $10.5 \%(n=2)$, and $-25 \pm 8 \%(n=2)$, respectively (Fig. $3 B)$. The mean results for all experiments recorded 2 min after the stimulation protocol are summarized in Fig. 4B. Examination of the test pulse EPSPs 10 min after the stimulation protocol, revealed that the depression seen when s. radiatum was stimulated out of phase with the s. lacunosum-moleculare was short-term, as shown in Fig. 4C. The test pulse EPSPs returned to baseline amplitudes within $10 \mathrm{~min}$ of the stimulation protocol (mean percentage EPSP change $=+1 \pm 3.5120 \mathrm{~ms}$ after burst start, $n=2 ;-3160 \mathrm{~ms}$ after burst start, $n=2 ; 0 \pm 2180$ ms after burst start, $n=2$ ).

As mentioned in the preceding text, stimulation of s. radiatum 15-100 ms after the start of the s. lacunosum-moleculare bursts, during the stimulation protocol, potentiated test pulse EPSPs recorded after $30 \mathrm{~min}$. However, examination of the test pulse EPSPs obtained from these preparations within 2 min of the stimulation protocol revealed that there was no significant change in the amplitude of the test pulse EPSPs (mean percentage EPSP change $=+2 \pm 3$ at $40 \mathrm{~ms}$ after burst start, $n=$ $6 ;-3.5 \pm 7.5$ at $70 \mathrm{~ms}$ after burst start, $n=2 ;-0.8 \pm 10.2$ at $100 \mathrm{~ms}$ after burst start, $n=2$; Fig. 4B). The test pulse EPSPs did not remain unchanged though and were potentiated within $10 \mathrm{~min}$ (Fig. 4C). S. radiatum pulses delivered 40, 70, and $100 \mathrm{~ms}$ after the start of the s. lacunosum-moleculare bursts, potentiated the test pulse EPSP by $+17 \pm 8 \%(n=6)$, $+19 \pm 10 \%(n=2)$, and $+15 \pm 3 \%(n=2)$, respectively. These results may indicate that the potentiation induced by stimulating $\mathrm{s}$. radiatum in phase with the s. lacunosum-moleculare is suppressed during the first few minutes of the test period by the short-term depression, which is unmasked when s. radiatum is stimulated out of phase with the s. lacunosummoleculare.

Interestingly, stimulation of s. radiatum $5 \mathrm{~ms}$ after the start of the s. lacunosum-moleculare bursts, during the stimulation protocol, caused a short-term depression of the test pulse EPSP amplitudes similar to that seen when s. radiatum was stimulated out of phase with the s. lacunosum-moleculare. The amplitude of test pulse EPSPs recorded 2 min after the stim- 

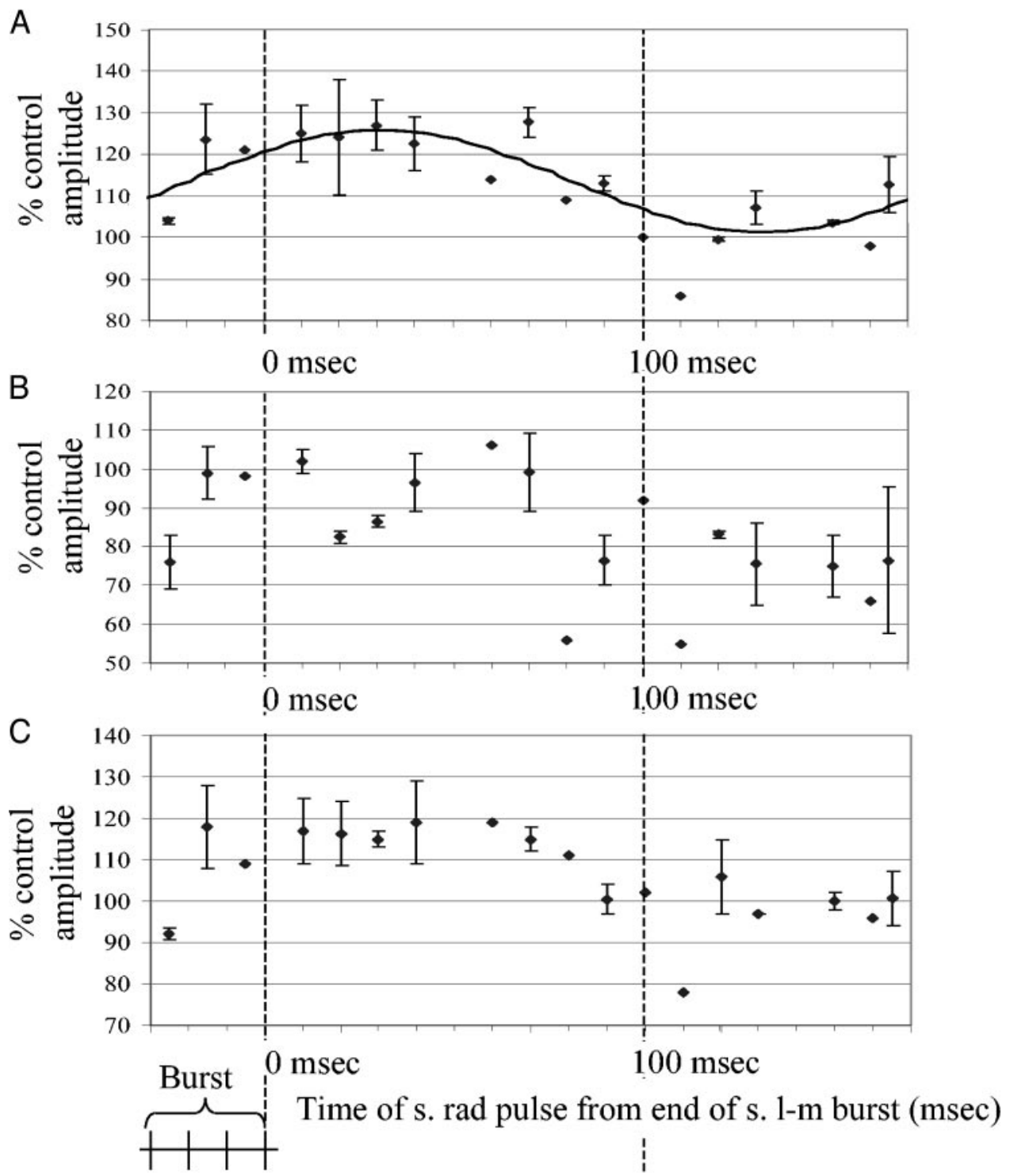

FIG. 4. Summary of the mean test pulse amplitudes obtained at different time points after the stimulation protocol. Test pulse amplitudes are plotted as a mean percentage of the control amplitude and are shown for each variation in the timing of the s. radiatum pulse in relation to the s. lacunosummoleculare bursts during the stimulation protocol. A: mean test pulse amplitudes obtained $30 \mathrm{~min}$ after the stimulation protocol. The test pulse amplitudes were potentiated when s. radiatum was stimulated within 70 $\mathrm{ms}$ of the end of the s. lacunosum-moleculare bursts during the stimulation protocol and were not significantly changed when $\mathrm{s}$. radiatum was stimulated $>70 \mathrm{~ms}$ after the end of the s. lacunosum-moleculare burst. The curve on the plot shows a sine wave fitted to the data points to determine the phase of best fit. This curve has an amplitude of $27 \%$, a frequency of $5 \mathrm{~Hz}$ and a peak at $58.6 \mathrm{~ms}$. This peak falls $28.6 \mathrm{~ms}\left(51.4^{\circ}\right)$ after the last pulse of the stimulation in s. lacunosum-moleculare. $B$ : mean test pulse amplitudes obtained $2 \mathrm{~min}$ after the stimulation protocol. The test pulse amplitudes were depressed when s. radiatum was stimulated $>70 \mathrm{~ms}$ after the start of the s. lacunosummoleculare bursts during the stimulation protocol. Note that $\mathrm{s}$. radiatum stimulation 5 ms after the start of the s. lacunosum-moleculare bursts during the stimulation protocol also depressed the test pulse amplitude. $C$ : mean test pulse amplitudes obtained 10 min after the stimulation protocol. The depression of test pulse amplitudes in preparations in which s. radiatum was stimulated $>70 \mathrm{~ms}$ after the end of the s. lacunosummoleculare bursts shown in $B$ is short-term as the test pulses returned to control amplitudes within 10 min of the stimulation protocol.

\section{DEGREES}

180 DEGREES ulation protocol had decreased by $-24 \pm 7 \%$ ( $n=4$; Fig. $4 B$ ), but the test pulse EPSPs returned to baseline amplitudes within $10 \min (-8 \pm 1.5 \%$; $n=4$; Fig. $4 \mathrm{C})$. This seems to indicate that the mechanism by which LTP is induced when s. radiatum is stimulated in phase with s. lacunosum-moleculare does not start until at least after the first $5 \mathrm{~ms}$ of the s. lacunosummoleculare bursts. This is consistent with the overall evidence that induction of LTP is phase delayed relative to the stimulation of entorhinal input fibers in s. lacunosum-moleculare.

\section{Control experiments}

Stimulation of s. radiatum alone with 10 single pulses at 5 $\mathrm{Hz}$, set at the same stimulus intensity used during the test period, did not cause a significant change in the test pulse EPSP amplitudes recorded $2 \mathrm{~min}(-1.2 \pm 0.6 \%), 10 \mathrm{~min}$ $(-2.3 \pm 1 \%)$, and $30 \mathrm{~min}(+3 \pm 1.3 \%$; Fig. 5), after the stimulation protocol $(n=6)$. Likewise, when s. lacunosummoleculare was stimulated alone with a strong $(200 \mu \mathrm{A})$ theta-burst pattern (10 bursts at $5 \mathrm{~Hz}, 4$ pulses per burst at 100
$\mathrm{Hz})$, the test pulse EPSP amplitudes recorded 2 min $(-7.7 \pm$ $4.5 \%), 10 \mathrm{~min}(+1 \pm 3.5 \%)$, and $30 \mathrm{~min}(+5 \pm 7.1 \%$; Fig. 5$)$, after the stimulation protocol did not change significantly $(n=4)$.

\section{Fitting LTP data to a sine wave function}

To determine the peak phase of induction of LTP, the experimental data on long-term potentiation at $30 \mathrm{~min}$ were fitted to a sine wave curve. As noted in Eq. 4, in the mathematical analysis presented here, we used a sine wave, which was algebraically shifted to be positive. Therefore for fitting the data at $30 \mathrm{~min}$, we used a sine wave function representing positive induction of LTP (starting at 100\% of baseline strength and increasing). We fit the curve using Eq. 4 with the $1 / 2$ constants modified to $m / 2$, where $m$ is the magnitude of the sine wave added to the baseline. The curve was constrained to have frequency of $5 \mathrm{~Hz}$. We used nonlinear regression in the GraphPad Prism software package to obtain the best fit and then confirmed the phase of best fit using computation of $\mathrm{R}^{2}$ in 


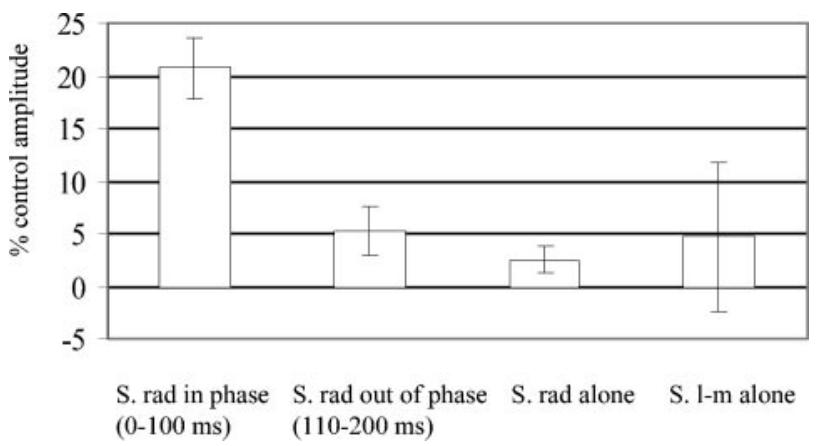

FIG. 5. Mean test pulse amplitudes obtained $30 \mathrm{~min}$ after the stimulation protocol in control preparations with stimulation of s. radiatum alone or $\mathrm{s}$. lacunosum-moleculare alone (right), compared with data combined for delays between 0 and $100 \mathrm{~ms}$ and between 110 and $200 \mathrm{~ms}$ (left). Stimulation of s. radiatum alone or s. lacunosum-moleculare alone did not cause a significant change in the test pulse amplitude. Error bars represent SE.

MATLAB. The curve obtained with curve fitting to the data are shown in Fig. 4. The fit yielded a curve with the amplitude $m=$ 27.0 and phase $=15.4^{\circ}(8.6 \mathrm{~ms})$. Note that a sine wave normally peaks at $90^{\circ}$, although in most of this paper, we have referred to the positive peak of the sine wave as $0^{\circ}$ in keeping with conventional descriptions of theta rhythm phase relative to the positive peak of an oscillation (Fox et al. 1986). Thus as can be seen in Fig. 4, the sine wave determined by curve fitting has been shifted from where it should normally peak at $90^{\circ}(50$ $\mathrm{ms})$ to a peak $\sim 58.6 \mathrm{~ms}\left(105.4^{\circ}\right)$. Note that a sine wave is very broad, so that the phase corresponds to the center of a distribution that shows significant induction of LTP at a wide range of phases.

As noted in the preceding text, in most parts of this paper, we will refer to the peak of the sine wave as $0^{\circ}$ instead of $90^{\circ}$ in keeping with conventions for describing the phase of physiological phenomena relative to the theta wave in the EEG. Now consider that we are comparing with entorhinal input represented by the stimulus pulses delivered at $0,10,20$, and $30 \mathrm{~ms}$. We will assume that this stimulation of s. lacunosummoleculare causes depolarization in the dendrites in s. radiatum that is somewhat delayed relative to the stimulation (due to the time constant of the dendritic membrane). We will also assume that temporal summation of the postsynaptic depolarization causes the peak of depolarization to occur just after the last pulse of the s. lacunosum-moleculare stimulation (at $30 \mathrm{~ms}$ ). Thus we will assume the rhythmic input to s. lacunosummoleculare has its peak at $30 \mathrm{~ms}$. Thus if we consider the peak of the EC sine wave to be at the end of the s. lacunosummoleculare burst at $30 \mathrm{~ms}$ and the peak of LTP induction to be at $58.6 \mathrm{~ms}$, then the peak of the sine wave fitted to the data on induction of LTP occurs at a phase delay of $28.6 \mathrm{~ms}\left(51.4^{\circ}\right)$ relative to the EC sine wave. This provides an approximate measure of the phase delay between the entorhinal input to $\mathrm{s}$. lacunosum-moleculare and the induction of LTP at CA3 input to $\mathrm{s}$. radiatum. This falls close to the phase difference of $30^{\circ}$ that provides the best function in the model. At $51.4^{\circ}$, the performance measure will be at $95.8 \%$ of its value at $30^{\circ}$. If we assume the peak of dendritic depolarization caused by $\mathrm{s}$. lacunosum-moleculare input is even later than the last input pulse, then this phase difference would be smaller. In these discussions, note that we have focused on the peak of dendritic depolarization caused by s. lacunosum-moleculare stimulation.
The maximal synaptic input to s. lacunosum-moleculare actually occurs at the trough of the theta rhythm EEG recorded in stratum lacunosum-moleculare of rats in vivo (Brankack et al. 1993).

To explore the statistical significance of this fit to the 39 experiments in the data, we compared the fit with the parameters described in the preceding text to fits with different amplitude or phase. Note that we started with an a priori hypothesis that the data would fit a sine wave function with wavelength $200 \mathrm{~ms}(5 \mathrm{~Hz})$. The fit described in the preceding text had an $R^{2}$ value of 0.39 . The $95 \%$ confidence interval for the amplitude of the sine wave was 7.8 to 19.3 , indicating the statistically significant superiority of the sine wave to a flat line (low-amplitude sine wave). A fit with amplitude constrained to absolute value $<1.0$ gave an $R^{2}=0.06$. Akaike's information criterion in Graphpad Prism gave a clear preference at $P<$ 0.05 for the sine wave with amplitude 27 versus amplitudes $<12$. In addition, analysis demonstrates the statistical significance of the phase of the curve fit. The $95 \%$ confidence interval for phase was $27.2-75.5^{\circ}(<1 / 6$ of the full range of possible phases). Akaike's information criterion in Graphpad gave a clear preference at $P<0.05$ for the sine wave with phase $51.4^{\circ}$ versus phases $<20$ or $>81^{\circ}$. Thus the data demonstrate with statistical significance at $P<0.05$ that the peak induction of LTP is at a phase lag $>20$ and $<81^{\circ}$ and that the modeled phase delay of $30^{\circ}$ falls within the $95 \%$ confidence range of the experimental data.

\section{I S C U S S I O N}

The experimental data presented here show a phase offset for the induction of LTP that appears consistent with the mathematical results on associative memory function in a model of theta rhythm interactions in the hippocampus. The maximal induction of LTP appears when CA3 input (s. radiatum stimulation) follows EC input (s. lacunosum-moleculare stimulation) at a close interval but not when the CA3 input is out of phase with the EC input. Fitting of a sine wave to the data on magnitude of LTP at 30 min (Fig. 4A) shows that the center of the phase of best LTP induction occurs at $\sim 28.6 \mathrm{~ms}$ after the last of the four pulses delivered to s. lacunosummoleculare. This corresponds to a phase delay of $\sim 51.4^{\circ}$ between the peak of $\mathrm{s}$. lacunosum-moleculare input and the peak of LTP induction. This falls close to the phase difference of $30^{\circ}$, which provides the best function in the model. At $51.4^{\circ}$, the performance measure will be at $95.8 \%$ of its value at $30^{\circ}$. Note that in both the model and the experiment, induction of LTP is strong at a wide range of phases. For example, in Fig. $4 A$ induction of LTP appears with phase delays between $-27^{\circ}$ $(-15 \mathrm{~ms})$ and $126^{\circ}(70 \mathrm{~ms})$. Thus the exact location of the peak is less important than the qualitative evidence for a phase delay between entorhinal input and LTP induction provided by the curve fitting of the data. The evidence for a phase delay in the physiological data is consistent with the requirement of a phase delay in the mathematical analysis.

The phase dependence of LTP described here could be important for the timing of encoding and retrieval dynamics within hippocampal networks. The mathematical modeling builds on previously published models (Hasselmo and Schnell 1994; Hasselmo et al. 2002a,b). Those previous papers started with simple models of the learning of associations between 
locations and reward necessary for performing spatial memory tasks. Incorporation of oscillatory dynamics in those models enhanced their function and demonstrated what phase relationships provided the best function. In previous models, the best function occurred with EC input and LTP induction at the same phase, but those previous models allowed LTD to cause forgetting during retrieval. In contrast, the modification of the model presented here assumes that induction of LTP is positive at all phases. This requires that interference between patterns be resolved by context-dependent retrieval rather than by forgetting of the initial association. The best memory function was evaluated with the performance measure presented in $E q$. 1 , which had its maximal value when LTP induction followed peak entorhinal input by $\sim 30^{\circ}$.

These models provide a functional framework for linking the specific phase dependence of physiological variables to the role of theta rhythm in behavior. In these models, the loss of theta rhythm would allow less effective encoding (Hasselmo et al. 2002a,b). This could account for the impairment of spatial memory and reversal learning caused by lesions which decrease theta rhythm (M'Harzi et al. 1987; Winson 1978). If encoding dynamics are best on a specific phase of theta, this could account for why theta rhythm appears to phase lock to the input of a visual stimulus in a delayed matching task (Givens 1996) and to the timing of sniffing in a reversal of olfactory discrimination (Macrides et al. 1982).

The physiological results presented here are consistent with the results from previous studies on LTP induction relative to theta in which s. radiatum was stimulated at the peak and/or trough of local CA1 theta (Huerta and Lisman 1995; Hölscher et al. 1997; Hyman et al. 2003). In those previous studies, LTP was induced when s. radiatum was stimulated at the peak of local CA1 theta, when EC input was strong but not when s. radiatum was stimulated at the trough of local CA1 theta when EC input was weak. The present results suggest the exact peak of local theta may not be the maximal point on the LTP induction function. Best induction of LTP might be slightly delayed relative to the peak of local theta in s. radiatum. The first demonstration that the induction of LTP depended on the phase of stimulation relative to theta was done on the perforant pathway input to dentate gyrus (Pavlides et al. 1988), and this was replicated in awake animals (Orr et al. 2001). The phase dependence of perforant path LTP could enhance associative function at synapses from the medial entorhinal cortex to the middle molecular layer of the dentate gyrus.

These results are also consistent with previous data from slice preparations using paired stimulation of s. radiatum and s. lacunosum-moleculare. Our study is novel because the induction of LTP by correlating s. radiatum and s. lacunosummoleculare stimulation (at an intensity and frequency ineffective at inducing LTP separately) has not been shown before. However, two previous studies have shown how the timing of s. lacunosum-moleculare stimulation can modulate the induction of LTP by theta burst stimulation of s. radiatum (Levy et al. 1998; Remondes and Schuman 2002). In those previous studies, the induction of LTP at Schaffer collateral-CA1 pyramidal neuron synapses is reduced when s. radiatum conditioning trains, which can induce LTP alone, are paired with s. lacunosum-moleculare conditioning trains (Levy et al. 1998; Remondes and Schuman 2002). However, these data are still consistent with our results, as LTP was still induced when s. radiatum and s. lacunosum-moleculare stimulation were paired in those studies (although it is significantly less than if $s$. radiatum was stimulated alone). Note that we used stimulation of s. radiatum that did not induce LTP in control experiments where the s. lacunosum-moleculare stimulation was omitted. Interestingly, in previous studies, when s. radiatum stimulation is delayed to $50 \mathrm{~ms}$ after the start of the s. lacunosummoleculare burst, LTP induction is not inhibited (Levy et al. 1998). This is comparable to the results presented in this paper that show that $\mathrm{s}$. radiatum stimulation $60 \mathrm{~ms}$ after the start of the s. lacunosum-moleculare burst during the stimulation protocol potentiates the test pulse EPSP by $27 \pm 6 \%$, whereas stimulation during the s. lacunosum-moleculare burst at $5 \mathrm{~ms}$ does not cause potentiation. The absence of potentiation at the 5-ms delay may result from the same mechanisms that cause an absence of LTP induced by s. radiatum trains that are presented at the start or middle of a long (10 pulse) train to s. lacunosummoleculare (Levy et al. 1998). Thus both the data presented here and the previous data from Levy et al. suggest the importance of the s. radiatum input arriving at a phase delay relative to s. lacunosum-moleculare stimulation.

Although there is a debate about whether the predominant influence of EC input on CA1 (caused by s. lacunosummoleculare stimulation) is primarily inhibitory or excitatory (see Levy et al. 1995; Soltesz and Jones 1995), there is a lot of evidence that at least a component of it is inhibitory (Colbert and Levy 1992; Dvorak-Carbone and Schuman 1999; Empson and Heinemann 1995; Enoki et al. 2001; Lambert et al. 1991; Remondes and Schuman 2002). Remondes and Schuman (2002) showed that in the presence of the $\mathrm{GABA}_{\mathrm{A}}$ antagonist bicuculline, s. lacunosum-moleculare stimulation did not inhibit LTP at Schaffer collateral-CA1 synapses and in fact s. lacunosum-moleculare stimulation in phase with $\mathrm{s}$. radiatum stimulation enhanced the LTP. Another study showed that the sublinear summation of EC and CA1 inputs is only completely blocked with the use of both $\mathrm{GABA}_{\mathrm{A}}$ and $\mathrm{GABA}_{\mathrm{B}}$ antagonists (Enoki et al. 2001). It is therefore possible that the inhibitory component of s. lacunosum-moleculare stimulation causes a certain level of inhibition in CA1 pyramidal cell activity via postsynaptic $\mathrm{GABA}_{\mathrm{A}}$ receptors and presynaptic and postsynaptic $\mathrm{GABA}_{\mathrm{B}}$ receptors and is independent of s. radiatum timing, thus resulting in less LTP when s. lacunosum-moleculare and s. radiatum are in phase and STD when they are out of phase as shown in this paper. In particular, $\mathrm{GABA}_{\mathrm{B}}$-dependent inhibition of region CA1 spiking responses has been shown to have a relatively slow time course (Dvorak-Carbone and Schuman 1999). This slow time course is consistent with the fact that STD in our study appeared in later portions of the 200-ms interval between EC stimulation trains.

It seems likely that LTP is induced when s. lacunosummoleculare stimulation is in phase with s. radiatum stimulation because s. lacunosum-moleculare stimulation depolarizes the dendrites of CA1 pyramidal neurons. S. lacunosum-moleculare stimulation generates dendritic spikes in CA1 pyramidal neurons; this leads to LTP without requiring postsynaptic action potentials (Golding et al. 2002). This is consistent with the data that demonstrate that at the peak of local theta at the time of the strongest EC input, CA1 pyramidal cell dendrites are depolarized but the somas are simultaneously hyperpolarized (Kamondi et al. 1998), resulting in less pyramidal cell spiking (Fox et al. 1986; Skaggs et al. 1996). The lack of LTP when s. radiatum is 
stimulated 110-195 ms after the start of the s. lacunosum-moleculare bursts during the stimulation protocol may result from the reduction of dendritic depolarization.

The small time window of $100 \mathrm{~ms}$, in which s. radiatum and s. lacunosum-moleculare have to be stimulated to induce LTP, is surprising when you consider LTP induction at Schaffer collateral-CA1 synapses is largely dependent on $N$-methyl-Daspartate (NMDA) receptors (Wantanabe et al. 2002), which have a deactivation rate of $\sim 200 \mathrm{~ms}$ (Spruston et al. 1995). However, the requirement of an even shorter time window for covariance of pre- and postsynaptic activity has been shown previously with dual stimulation in s. radiatum of CA1 (Stanton 1996; Stanton and Sejnowski 1989). Several other studies that have shown LTP induced by pairing two stimuli requires that spiking induced in pre- and postsynaptic neurons should be separated by < $100 \mathrm{~ms}$ (Bi and Poo 1998; Debanne et al. 1998; Levy and Steward 1983; Markam et al. 1997). Thus the interaction of s. lacunosum-moleculare with s. radiatum input may have a slightly slower time course than the timing associated with direct somatic current injection or with stimulation of different inputs in the same layer (Stanton and Sejnowski 1989). It is thought that correlated stimuli boost the amplitude of the dendritic spikes and unblock the NMDA receptors and induce LTP. Possible mechanisms for dendritic spike boosting include the activation of dendritic $\mathrm{Na}^{+}$channels (Stuart and Hausser 2001) and the inactivation of A-type $\mathrm{K}^{+}$channels (Wantanabe et al. 2002). Another possible mechanism that is not mutually exclusive from the preceding two is that there is a cooperative relationship between $\mathrm{Ca}^{2+}$ and the $\mathrm{Ca}^{2+}$ binding site involved in LTP induction (Bi and Poo 1998).

In summary, the mathematical analysis presented here demonstrates the potential importance for associative memory function of a phase delay between entorhinal input in $\mathrm{s}$. lacunosum-moleculare and the induction of LTP in s. radiatum. Physiological data presented here demonstrate that the induction of LTP does appear at a phase delay relative to stimulation of entorhinal input in s. lacunosum-moleculare.

\section{G R A N T S}

This work was supported by National Institutes of Health Grants MH60013, MH-61492, MH-60450, and DA-16454

\section{REFERENCES}

Amaral DG and Witter MP. The three-dimensional organization of the hippocampal formation: a review of anatomical data. Neuroscience 31: 571-591, 1989.

Berry SD and Thompson RF. Prediction of learning rate from the hippocampal electroencephalogram. Science 200: 1298-1300, 1978.

Bi G and Poo M. Synaptic modifications in cultured hippocampal neurons: dependence on spike timing, synaptic strength, and postsynaptic cell type. J Neurosci 18: 10464-10472, 1998.

Bland BH. The physiology and pharmacology of hippocampal-formation theta rhythms. Prog Neurobiol 25: 1-54, 1986.

Bragin A, Jando G, Nadasdy Z, Hetke J, Wise K, and Buzsáki G. Gamma $(40-100 \mathrm{~Hz})$ oscillation in the hippocampus of the behaving rat. $J$ Neurosci 15: 47-60, 1995.

Brankack J, Stewart M, and Fox SE. Current source density analysis of the hippocampal theta rhythm: associated sustained potentials and candidate synaptic generators. Brain Res 615: 310-327, 1993.

Buzsáki G. Theta oscillations in the hippocampus. Neuron 33: 325-340, 2002.

Buzsáki G, Czopf J, Kondakor I, and Kellenyi L. Laminar distribution of hippocampal rhythmic slow activity (RSA) in the behaving rat: currentsource density analysis, effects of urethan and atropine. Brain Res 365: 125-137, 1986.
Chrobak JJ and Buzsáki G. Gamma oscillations in the entorhinal cortex of the freely behaving rat. J Neurosci 18: 388-398, 1998.

Chrobak JJ, Lorincz A, and Buzsáki G. Physiological patterns in the hippocampo-entorhinal cortex system. Hippocampus 10: 457-465, 2000.

Colbert CM and Levy WB. Electrophysiological and pharmacological characterization of perforant path synapses in CA1: mediation by glutamate receptors. J Neurophysiol 68: 1-8, 1992.

Debanne D, Gahwiler BH, and Thompson SM. Long-term synaptic plasticity between pairs of individual CA3 pyramidal cells in rat hippocampal slice cultures. J Physiol 507: 237-247, 1998.

Dvorak-Carbone H, and Schuman EM. Patterned activity in stratum lacunosum moleculare inhibits CA1 pyramidal neuron firing. J Neurophysiol 81: 1036-1044, 1999.

Empson RM and Heinemann U. The perforant path projection to hippocampal area CA1 in the rat hippocampal-entorhinal cortex combined slice. J Physiol 484: 707-720, 1995.

Enoki R, Inoue M, Hashimoto Y, Kudo Y, and Miyakawa H. GABAergic control of synaptic summation in hippocampal CA1 pyramidal neurons. Hippocampus 11: 683-689, 2001.

Fox SE. Membrane potential and impedence changes in hippocampal pyramidal cells during theta rhythm. Exp Brain Res 77: 283-294, 1989.

Fox SE, Wolfson S, and Ranck JBJ. Hippocampal theta rhythm and the firing of neurons in walking and urethan-anesthetized rats. Exp Brain Res 62: 495-508, 1986.

Givens B. Stimulus-evoked resetting of the dentate theta rhythm: relation to working memory. Neuroreport 8: 159-163, 1996.

Golding NL, Staff NP, and Spruston N. Dendritic spikes as a mechanism for cooperative long-term potentiation. Nature 418: 326-331, 2002.

Green JD and Arduini AA. Hippocampal electrical activity and arousal. J Neurophysiol 15: 533-577, 1954.

Griffin AL, Asaka Y, Darling RD, and Berry SD. Theta-contingent trial presentation accelerates learning rate and enhances hippocampal plasticity during trace eyeblink conditioning. Behav Neurosci 118: 403-411, 2004.

Hasselmo ME, Bodelón C, and Wyble BP. A proposed function for hippocampal theta rhythm: separate phases of encoding and retrieval enhance reversal of prior learning. Neural Comput 14: 793-817, 2002a.

Hasselmo ME, Hay J, Ilyn M, and Gorchetchnikov A. Neuromodulation, theta rhythm and rat spatial navigation. Neural Networks 15: 689-707, 2002b.

Hasselmo ME and Schnell E. Laminar selectivity of the cholinergic suppression of synaptic transmission in rat hippocampal region CA1: Computational modeling and brain slice physiology. J Neurosci 14: 3898-3914, 1994

Hölscher C, Anwyl R, and Rowan MJ. Stimulation on the positive phase of hippocampal theta rhythm induces long-term potentiation that can be depotentiated by stimulation on the negative phase in area CA1 in vivo. J Neurosci 17: 6470-6477, 1997.

Huerta PT and Lisman JE. Bidirectional synaptic plasticity induced by a single burst during cholinergic theta oscillation in CA1 in vitro. Neuron 15: 1053-1063, 1995.

Hyman JM, Wyble BP, Goyal V, Rossi CA, and Hasselmo ME. Stimulation in hippocampal region CA1 in behaving rats yields LTP when delivered to the peak of theta and LTD when delivered to the trough. J Neurosci 23: 11725-11731, 2003.

Judge SJ and Hasselmo ME. Induction of LTP in stratum radiatum during theta rhythm stimulation of stratum lacunosum-moleculare in rat hippocampus. Soc Neurosci Abstr 80.7, 2002.

Kamondi A, Acsády L, Wang XJ, and Buzsáki G. Theta oscillations in somata and dendrites of hippocampal pyramidal cells in vivo: activitydependent phase-precession of action potentials. Hippocampus 8: 244-261, 1998.

Lambert NA, Borroni AM, Grover LM, and Teyler TJ. Hyperpolarizing and depolarizing $\mathrm{GABA}_{\mathrm{A}}$ receptor-mediated dendritic inhibition in area CA1 of the rat hippocampus. J Neurophysiol 66: 1538-1548, 1991.

Levy WB, Colbert CM, and Desmond NL. Another network model bites the dust: Entorhinal inputs are no more than weakly excitatory in the hippocampal region. Hippocampus 5: 137-140, 1995.

Levy WB, Desmond NL, and Zhang DX. Perforant path activation modulates the induction of long-term potentiation of the Schaffer collateral-hippocampal CA1 response: theoretical and experimental analyses. Learn Mem 4: 510-518, 1998.

Levy WB and Steward O. Temporal continguity requirements for long-term associative potentiation/depression in the hippocampus. Neuroscience 8: 791-797, 1983. 
Macrides F, Eichenbaum HB, and Forbes WB. Temporal relationship between sniffing and the limbic theta rhythm during odor discrimination reversal learning. J Neurosci 2: 1705-1717, 1982.

Markram H, Lubke J, Frotscher M, and Sakmann B. Regulation of synaptic efficacy by coincidence of postsynaptic APs and EPSPs. Science 275: 213-215, 1997.

M'Harzi M, Palacios A, Monmaur P, Willig F, Houcine O, and Delacour J. Effects of selective lesions of fimbria-fornix on learning set in the rat. Physiol Behav 40: 181-188, 1987.

Orr G, Rao G, Houston FP, McNaughton BL, and Barnes CA. Hippocampal synaptic plasticity is modulated by theta rhythm in the fascia dentate of adult and aged freely behaving rats. Hippocampus 11: 647-654, 2001.

Pavlides C, Greenstein YJ, Grudman M, and Winson J. Long-term potentiation in the dentate gyrus is induced preferentially on the positive phase of theta-rhythm. Brain Res 439: 383-387, 1988.

Remondes $M$ and Schuman EM. Direct cortical input modulates plasticity and spiking in CA1 pyramidal neurons. Nature 416: 736-740, 2002.

Seager MA, Johnson LD, Chabot ES, Asaka Y, and Berry SD. Oscillatory brain states and learning: impact of hippocampal theta-contingent training. Proc Natl Acad Sci USA 99: 1616-1620, 2002.

Skaggs WE, McNaughton BL, Wilson MA, and Barnes CA. Theta phase precession in hippocampal neuronal populations and the compression of temporal sequences. Hippocampus 6: 149-172, 1996.
Soltesz I and Jones RS. The direct perforant path input to CA1: excitatory or inhibitory? Hippocampus 5: 101-103, 1995.

Spruston N, Jonas P, and Sakmann B. Dendritic glutamate receptor channelsin rat hippocampal CA3 and CA1 pyramidal neurons. J Physiol 482: 325-352, 1995.

Stanton PK. LTD, LTP, and the sliding threshold for long-term synaptic plasticity. Hippocampus 6: 35-42, 1996.

Stanton PK and Sejnowski TJ. Associative long-term depression in the hippocampus induced by Hebbian covariance. Nature 339: 215-218, 1989.

Stuart GJ and Hausser M. Dendritic coincidence detection of EPSPs and action potentials. Nat Neurosci 4: 63-71, 2001.

Watanabe S, Hoffman DA, Migliore M, and Johnston D. Dendritic $\mathrm{K}^{+}$ channels contribute to spike-timing dependent long-term potentiation in hippocampal pyramidal neurons. Proc Natl Acad Sci USA 99: 8366-8371, 2002.

Winson J. Loss of hippocampal theta rhythm results in spatial memory deficit in the rat. Science 201: 160-163, 1978.

Witter MP, Griffioen AW, Jorritsma-Byham B, and Krijnen JLM. Entorhinal projections to the hippocampal CA1 region in the rat: an underestimated pathway. Neurosci Lett 85: 193-198, 1988.

Wyble BP, Hyman JM, Goyal V, and Hasselmo ME. Phase relationship of LTP induction and behavior to theta rhythm in the rat hippocampus. Soc Neurosci Abstr 537.19, 2001. 